www.jmscr.igmpublication.org

Impact Factor (SJIF): 6.379

Index Copernicus Value: 71.58

ISSN (e)-2347-176x ISSN (p) 2455-0450

crossref DOI: _https://dx.doi.org/10.18535/jmscr/v6i4.69

Journal Of Medical Science And Clinical Research

\title{
Randomised Controlled Study of Short Term Outcomes of Laparoscopic Inguinal Hernia Mesh Repair and Lichensteins Inguinal Hernia Mesh Repair
}

\author{
Authors \\ Dr Santhosh Kumar $\mathbf{R}^{1}$, Dr Renjin R $\mathbf{P}^{2}$, Dr Toney Jose ${ }^{3}$, Dr Santhosh Urs ${ }^{4}$ \\ ${ }^{1}$ Associate Professor, Dept of General Surgery, Govt Medical College, Kottayam \\ ${ }^{2}$ Assistant Professor, Dept of General Surgery, Govt Medical College, Kottyam \\ ${ }^{3,4}$ Junior Resident, Dept of General Surgery, Govt Medical College, Kottayam
}

\begin{abstract}
Inguinal hernia surgery is a common surgery being performed all over the world. With the advent of laparoscopy, it has also been used in inguinal hernia surgery. Laparoscopy is associated with faster recovery and lower wound complications as compared to open surgery. This study aimed to compare laparoscopy with open repair for inguinal hernia. 60 patients with inguinal hernia were randomised to open and laparoscopic procedures with 30 patients in each group. The duration of surgery, post operative complication, duration of hospital stay and time to return to work were analysed. The mean operating duration was 42.03 +/-6.5 min in open and 84.97+/-13.9 in laparoscopy group. Among the 30 cases that underwent open mesh repair, 3 cases required urethral catheterization for urinary retention, 3 cases suffered chronic pain, 2 cases had wound related complications and 1 had wound infection. The total complication rate of the open group was $30 \%$. Among the 30 cases that underwent laparoscopic repair, only 1 required urethral catheterization for urinary retention and 2 cases had wound related complications, total complication rate for this group being 10\%. The mean duration of hospital stay was 1.17+/-0.3 days for laparoscopy group and 2.5+/-0.5 days for open group. The time to return to work was 5.67+/-1.3 days for laparoscopy and 17.37+/-2.5 for open. Thus the use of laparoscopy in inguinal hernia surgery results in faster recovery and reduced complications as compared to open surgery.
\end{abstract}

Keywords: Laparoscopy, Hernia, Inguinal, Hernioplasty, Mesh repair.

\section{Introduction}

Hernia is among the oldest known afflictions of humankind, and surgical repair of the inguinal hernia is one of the most common general surgery procedure performed today. Despite the high incidence, the technical aspects of hernia repair continue to evolve ${ }^{(1)}$.

The treatment of inguinal hernias is integral to the history and current status of general surgery; evolution in the treatment of inguinal hernias has paralleled technologic developments in the field. The most significant advances to impact inguinal hernia repair have been the addition of prosthetic materials to conventional repairs and the introduction of laparoscopy to general surgical procedures.

With the advent of minimally invasive surgery, inguinal hernia repair underwent its most recent 
transformation. Laparoscopic inguinal hernia repair has added to the armamentarium of the general surgeon, gaining its popularity by providing a technique that lessens postoperative pain and improves recovery. Furthermore, an array of prosthetic materials have and are been introduced to further lower recurrence rates and provide the patient with the utmost quality of life. With the new found love for laparoscopy, studies to evaluate and compare the safety and usefulness of laparoscopy in inguinal hernia show advantage for laparoscopy. The laparoscopic technique, however, requires general anesthesia, and it is more often associated with serious intraoperative complications than is open repair although such complications are infrequent. The current state of surgical treatment of inguinal hernia depends on a sound foundation of the inguinal anatomy. The application of current technologies to this anatomic knowledge has fostered successful treatment of inguinal hernias with minimal morbidity heretofore unknown to surgical practice $^{(2)}$.

Inguinal hernia repair is one of the surgeries performed on a very regular basis. This study aims at studying the efficiency, advantages, disadvantages, limitations, post operative course and duration of hospital stay involved in open inguinal hernia mesh repair and laparoscopic inguinal hernia mesh repair surgeries and to arrive at a conclusion as to the best modality of treatment after comparison of morbidity of these procedures among them and in relation to standard published material.

\section{Methods}

This randomised control trial was carried out in the Department of General Surgery of our institution from March 2015 to August 2015. 60 cases of primary inguinal hernia were selected for the study. Permission of ethical committee and informed consent of each patient was taken. Patients with clinical evidence of inguinal hernia were admitted and were subjected to full history and examination, routine investigations like complete blood count, blood sugar level, serum creatinine, chest X-ray, ECG etc. Additional investigations like abdominal ultrasonography, CT scan of abdomen were done in cases with equivocal findings and suspected of other pathologies. Patients aged 15 to 75 years with unilateral or bilateral primary inguinal hernia planned for elective repair were included in the study. Patients with recurrent hernia were excluded from the study. The type of anaesthesia used was spinal anaesthesia for open cases and general anaesthesia for laparoscopic hernia mesh repair. The patients were randomized according to their serial number to undergo open or laparoscopic hernia mesh repair. All cases with a odd serial number underwent laparoscopic repair (TEP, TAPP) and all cases with an even serial number underwent open mesh repair. A single dose of preoperative broad spectrum antibiotic was given followed by the same postoperatively. Analgesics included Inj tramadol and Inj paracetamol postoperatively for 1 day and oral analgesics were continued thereafter. After surgery all patients were monitored carefully for pain, bleeding, wound infection and urinary retention. Wound infection was graded from minimal discharge of serous/pus from a single cutaneous suture to extensive and invasive process requiring hospitalization and intravenous antibiotics. Bleeding was defined as subcutaneous hematoma. Urinary retention was defined as inability to void requiring catheterization.The patients were discharged when fit and were asked to come for follow-up after 7 days, 1 and 3 months postoperatively. The patients were advised to return to their previous lifestyle except lifting heavy weights. Statistical analysis was carried out using SPSS.

\section{Results}

The study included 60 patients (59 male and 1 female) with inguinal hernia. 30 patients underwent laparoscopic hernia repair and 30 underwent open Lichtenstein hernia repair. There was no difference in age among the groups. Mean 
age was $44.7+/-15.55$ in open repair and $46.8+/-$ 15.6 in laparoscopic repair. Majority of patients were between 40 and 60 years of age in both groups. $33.2 \%$ and $46.6 \%$ in open and laparoscopic groups respectively. $70 \%$ of the cases presented with the swelling confined to the inguinal region whereas the remaining 30\% presented with an inguinoscrotal swelling. $50 \%$ of the cases enrolled in this study presented with a swelling within 1 to 6 months of its onset. Around $23 \%$ came within 6 to 12 months and around 17\% presented after 1 year of the onset of swelling. 49 patients had right sided hernia and 11 had left sided hernia. Bilateral hernia was not noted in this study population. 43 patients had indirect and 17 patients had direct hernia.

Table 1: Operating time for open repair and laparoscopic repair

\begin{tabular}{|l|c|c|c|}
\hline $\begin{array}{l}\text { DURATION OF OPERATING } \\
\text { TIME (IN MINS) }\end{array}$ & $\begin{array}{c}\text { OPEN } \\
\text { REPAIR }\end{array}$ & $\begin{array}{c}\text { LAPAROSCOPIC } \\
\text { REPAIR }\end{array}$ & P VALUE \\
\hline MIN - MAX & $30-56$ & $67-107$ & \\
\cline { 1 - 3 } & & & \\
MEAN \pm S.D & $42.03 \pm 6.51$ & $84.97 \pm 13.97$ & $<0.001$ \\
\hline
\end{tabular}

In the open repair group, the minimum time of operation was 30 minutes and maximum was 56 minutes with a mean of 42.03 and a SD of 6.51. In the laparoscopic group, the minimum and maximum operating time was seen to be 67 and 107 minutes respectively with a mean of 84.97 and a SD of 13.97, with the $p$ value $<0.001$ (Table1).

Table 2: Complication following hernia repair

\begin{tabular}{|l|c|c|c|c|}
\hline \multirow{2}{*}{ POST OPERATIVE COMPLICATIONS } & \multicolumn{2}{|c|}{ OPEN REPAIR } & \multicolumn{2}{c|}{ LAPAROSCOPIC REPAIR } \\
\cline { 2 - 5 } URINARY RETENTION & NO. & $\%$ & NO. & $\%$ \\
\hline WOUND RELATED COMPLICATIONS & $\mathbf{3}$ & $\mathbf{1 0}$ & $\mathbf{1}$ & 3.33 \\
\hline CHRONIC PAIN & $\mathbf{2}$ & $\mathbf{6 . 6 7}$ & $\mathbf{2}$ & $\mathbf{6 . 6 7}$ \\
\hline WOUND INFECTION & $\mathbf{3}$ & $\mathbf{1 0}$ & - & - \\
\hline LUNG RELATED COMPLICATIONS & $\mathbf{1}$ & $\mathbf{3 . 3 3}$ & - & - \\
\hline RECURRENCE & - & - & - & - \\
\hline
\end{tabular}

Among the 30 cases that underwent open mesh repair, 3 cases required urethral catheterization for urinary retention, 3 cases suffered chronic pain, 2 cases had wound related complications and 1 had wound infection. The total complication rate of the open group was $30 \%$. Among the 30 cases that underwent laparoscopic repair, only 1 required urethral catheterization for urinary retention and 2 cases had wound related complications, total complication rate for this group being $10 \%$. (Table 2)

Table 3: Time to return to work

\begin{tabular}{|l|l|l|l|}
\hline RETURN TO WORK (IN DAYS) & OPEN REPAIR & LAPAROSCOPIC REPAIR & P VALUE \\
\hline MIN - MAX & $14-22$ & $3-8$ & $<0.001$ \\
\hline MEAN + S.D & $17.37 \pm 2.59$ & $5.67 \pm 1.35$ & $<$ \\
\hline
\end{tabular}

The minimum and maximum time taken to return to work in the open repair group were seen to be 14 and 22 days respectively with a mean of 17.37 and a SD of 2.59, whereas in the laparoscopic group the minimum was 3 days and maximum was 8 days, with a mean of 5.67 and a SD of 1.35 with $\mathrm{p}<0.001$. 
Table 4: Duration of hospital stay

\begin{tabular}{|l|l|l|l|}
\hline $\begin{array}{l}\text { DURATION OF HOSPITAL STAY } \\
\text { AFTER OPERATION (IN DAYS) }\end{array}$ & OPEN REPAIR & $\begin{array}{l}\text { LAPAROSCOPIC } \\
\text { REPAIR }\end{array}$ & P VALUE \\
\hline MIN - MAX & $2-4$ & $1-2$ & $<$ \\
\hline MEAN \pm S.D & $2.5 \pm 0.57$ & $1.17 \pm 0.38$ & $<0.05$ \\
\hline
\end{tabular}

The duration of stay in the hospital after operation ranged between 2 to 4 days in the open mesh repair group with a mean of 2.5 and a SD of 0.57 , whereas the duration of stay in the hospital after the laparoscopic procedure was between 1 to 2 days with a mean of 1.17 and a SD of 0.38 .

\section{Discussion}

Surgical repair of inguinal hernias is a common procedure in adult men. However, recurrence of hernias has been reported to occur after repair in 15 percent or more cases, and postoperative pain and disability are frequent. A laparoscopic method of performing a tension-free repair has been reported to result in low recurrence rates and to be associated with substantially less pain in the immediate postoperative period and earlier return to normal activities than the open-repair technique.

This study was conducted to compare the open mesh repair with the laparoscopic repair for inguinal hernia. In our study, $70 \%$ cases $(n=42)$ presented with a swelling confined to the inguinal region and $30 \%$ cases $(n=18)$ presented with a inguinoscrotal swelling.

In a study done by Khetri $\mathrm{R}$ et al. of 40 cases diagnosed with inguinal hernia in a hospital in Odisha, India, he observed that 37 cases had a swelling confined to the inguinal region and 3 cases presented with a inguinoscrotal swelling ${ }^{(3)}$.

Bhola Singh et al. reported that almost $56 \%$ of the cases studied presented with a swelling within a time period of 6 months to 1 year ${ }^{(4)}$.

Majority (50\%) of the cases in our study presented within 1 to 6 months duration and got operated. It is comparable to the previous study.

In a study by Dabbas et al., inguinal hernias were found to be more common on the right side than the left ${ }^{(5)}$. Similar results were noted by Delvin et al., $55 \%$ of the cases with inguinal hernia were on the right side with the remaining $45 \%$ on the left ${ }^{(6)}$. Mukesh $\mathrm{S}$ et al. from Northern India, found out $67 \%$ of the total inguinal hernia cases studied occurred on the right side and $30 \%$ on the left side (7).

In our study, we found that $81.67 \%$ of the cases presented with a right sided inguinal hernia whereas the remaining $18.33 \%$ presented with a left sided inguinal hernia with no cases of bilateral inguinal hernia noted. Our results are comparable to the above stated studies and we conclude that inguinal hernia does occur more commonly on the right side than the left.

Prior MJ et al., in his study on inguinal hernia reported that $60 \%$ of the cases presented with an indirect inguinal hernia and the remaining $40 \%$ had a direct hernia ${ }^{(8)}$. C Palanivelu et al. published his study on inguinal hernia and observed that $76 \%$ of the cases enrolled had an indirect inguinal hernia and the rest $24 \%$ had a direct hernia ${ }^{(9)}$. Similar results were obtained in our study. Milosevic P et al. observed that $67 \%$ of the hernias in his study were indirect and the remaining $33 \%$ were seen to be direct (10). Thus, indirect inguinal hernia is more common than direct inguinal hernia.

Zieran $\mathrm{J}$ et al. published his study and noted a mean operating time of $61 \pm 12$ minutes in the laparoscopic group and $36 \pm 14$ minutes in the open hernioplasty group ${ }^{(11)}$. B Johansson et al. noted similar results with a mean operating time of $65 \pm 25$ minutes for laparoscopic hernia repairs and $38 \pm 14$ minutes for open mesh repairs ${ }^{(12)}$. Pawanindra Lal and his colleagues, they recorded a mean operating time of $75.72 \pm 31.6$ minutes in the laparoscopic group and $54 \pm 15$ minutes in the open mesh repair group ${ }^{(13)}$. In a study done by the MRC Lap Groin Hernia Trial Group, the mean operating time for laparoscopic repair was 58.4 minutes and 43.3 minutes for open repair ${ }^{(14)}$. 
Picchio et al., in his study, reported a mean operating time of 49.6 and 33.9 minutes in the laparoscopic and open mesh repair techniques respectively ${ }^{(15)}$. In our study, the mean operating time in the laparoscopic group was $84.97 \pm 13.97$ minutes. In the open mesh repair group, the mean operating time was $42.03 \pm 6.51$ minutes with an extremely significant $\mathrm{p}$ value. The operating times for both the procedures are comparable to the above stated studies. Thus seen, laparoscopy requires a longer operating time than the open procedure.

In a study done on the outcomes of open mesh repair by Shaikh et al., $90.7 \%$ of the patients had uneventful recovery and 5\% developed surgical site infection ${ }^{(16)}$. Gianetta et al. in his study on open anterior mesh repair reported $2.7 \%$ scrotal hematoma, $2 \%$ cord edema, $0.7 \%$ orchitis and $0.7 \%$ wound infection ${ }^{(17)}$. Neumayer L et al., in his study found out the rate of complications to be higher in the laparoscopic-surgery group (39\%) than in the open-surgery group (33.4\%). The laparoscopic-surgery group had less pain initially than the open-surgery group on the day of surgery and at two weeks ${ }^{(18)}$. In a study comparing open and laparoscopic inguinal hernia repairs, Juul P et al. concluded that the complication rates in the two groups were similar ${ }^{(19)}$. In a study conducted by Bringman et al., the total complication rate in the laparoscopic group was seen to be $9.8 \%$ (wound hematoma 3.3\%, seroma and superficial infection $1.1 \%$ each, urinary retention $2.2 \%$, wound secretion and respiratory complication in $1.1 \%$ each) and in the open mesh group it was seen to be $20.4 \%$ (wound hematoma $7.8 \%$, superficial infection $3.9 \%$, prolonged pain and testicular swelling $1.9 \%$ each, wound secretion $2.9 \%$ and sensory loss $1.9 \%)^{(20)}$. Bhandarkar S et al., in his study reported that the wound infection rates were significantly lower after laparoscopic techniques (1\%) than after the Lichtenstein operation $(2.7 \%)$ and other open mesh repairs $(2.4 \%)$. The incidence of inguinal hematoma was found to be significantly lower after the laparoscopic repairs (13.1\%) than after the
Lichtenstein repair $(16.0 \%)$ as well as with the other open mesh techniques $(14.3 \%)^{(21)}$. Erhan et al. conducted a study and reported $4-6 \%$ of patients having chronic pain after Lichtenstein and preperitoneal hernia repair ${ }^{(22)}$ and Poobalan et al. reported a $10 \%$ incidence of chronic pain after open inguinal hernia repair ${ }^{(23)}$. In our study, among the 30 cases that underwent open mesh repair, $10 \%$ cases required urethral catheterization for urinary retention, $10 \%$ cases suffered chronic pain, $6.67 \%$ cases had wound related complications and $3.33 \%$ had wound infection. The total complication rate of the open group was $30 \%$. Among the 30 cases that underwent laparoscopic repair, $3.33 \%$ required urethral catheterization for urinary retention and $6.67 \%$ cases had wound related complications, total complication rate for this group being $10 \%$ and is comparable to the above stated studies. Laparoscopic surgery is this associated with lower complication rates when compared to open surgical procedures.

When return to work was addressed, Heikkinen et al. reported a mean period to return to normal life as 14 days in the laparoscopic group and 21 days in the open group ${ }^{(24)}$. In a study published by Wilson MS et al., the return to work was shorter in patients receiving laparoscopic repair (median 7 and 10 days, respectively) than Lichtenstein repair (14 and 21 days $)^{(25)}$. Mike Liem et al. observed the mean time to return to work in patients undergoing inguinal hernia repair and found it to be 14 and 21 days respectively for laparoscopic and open mesh repair groups ${ }^{(26)}$. Pawanindra Lal and his colleagues, the time until return to work was significantly lower in the laparoscopic group $(12.8 \pm 7$ days $)$ than in the open group $(19.3 \pm 4$ days $)^{(13)}$. Andersson et al., in his study, reported the mean time to return to work after the procedure to be 8 days in the laparoscopic group and 11 days in the open repair group ${ }^{(27)}$. In a study done by Stoker DL et al., the mean time for patients to return to their work post procedure was seen to be 14 days in the laparoscopic group and 28 days in the open repair group ${ }^{(28)}$. In our study, 
the mean time to return to work in the laparoscopic repair group was seen to be $5.67 \pm$ 1.35 days and $17.37 \pm 2.59$ days in the open mesh repair group with an extremely significant $\mathrm{p}$ value, and is comparable to the above stated studies, suggesting that return to normal daily activities and work is much earlier following a laparoscopic procedure for inguinal hernia repair.

Feliu X et al. observed a mean hospital stay of 0.6 \pm 0.8 days in the laparoscopic repair group and 1.3 \pm 1.2 days in the open repair group ${ }^{(29)}$. In various studies, like those conducted by Gokalp et al. ${ }^{(30)}$, Langeveld et al. ${ }^{(31)}$ and Eklund et al. ${ }^{(32)}$, all reported data on time to discharge favoring laparoscopic repair, however, none showed a significant difference between the laparoscopic group and the open repair group. In our study, the mean duration of hospital stay after a laparoscopic hernia repair was seen to be $1.17 \pm 0.38$ days and $2.5 \pm 0.57$ days following open mesh repair, with an extremely significant $p$ value and is comparable to the above stated studies suggesting a shorter hospital stay following a laparoscopic hernia repair than open mesh repair.

\section{Conclusion}

The surgical techniques for inguinal hernia continue to evolve. With the advent of laparoscopy, the technique of laparoscopic tension free repair is commonly practiced. The laparoscopic technique is associated with faster recovery and short hospital stay and early return to work with attendant increase in cost and difficult access. Studies have found that laparoscopic technique is a valid procedure to be performed in selected patients with inguinal hernia. Our study also found that laparoscopy is associated with faster recovery and lesser wound complications when compared to open surgery. With time, laparoscopy might become the standard of care in inguinal hernia surgery.

\section{References}

1. Patrick Javid, Jacob Greenberg, David C Brooks; Hernias. Maingot's Abdominal
Operations 12th ed. McGraw Hill, 2007; p.123-158.

2. Vadim Sherman, James R. Macho, F. Charles Brunicardi. Inguinal Hernias. Schwartz's Principles Of Surgery. $9^{\text {th }}$ ed. p. 1305-1343.

3. Khetri R, Dugar D, Ghata S, Comparative Study of Open Versus Laparoscopic Inguinal Hernia Repair. J Pharm Biomed Sci. 2014; 04(02): 113-117.

4. Bhola Singh Sidhu., et al 1999. "Tension free hernioplasty under local anesthesia 'Gilberts repair' “ Ind J Surg Vol 61 : 310314.

5. Dabbas N, Adams K, Pearson K, Royle G. Frequency of abdominal wall hernias: is classical teaching out of date? JRSM Short Rep. 2011;2(1):5.

6. Burke JB and Taylor M, "Clinical and economic effects of early return to work after inguinal hernia repair" $\mathrm{Br} \mathrm{J}$ Surg 1978; 65: 728-731.

7. Mukesh Sangwan, Vijayata Sangwan, Mahender Garg, Parveen Mahendirutta, Uma Garg, Abdominal wall hernia in a rural population in India-Is spectrum changing? Open Journal of Epidemiology, 2013, 3, 135-138.

8. M. J. PRIOR, E. V. WILliAMS, H. S. SHUKLA, S. PHILLIPS, S. VIG AND M. LEWIS, Prospective randomized controlled trial comparing Lichtenstein with modified Bassini repair of inguinal hernia. J R. Coll. Surg. Edinb., 43, April 1998, 82-86.

9. Palanivelu $\mathrm{C}$ et al. : 'Results of hand sutured laparoscopic hernioplasty' Effective method of repair. Ind J Surg 2000. 62(5); 339-341.

10. Milosevic, Predrag, and Marija Kolinovic. "Surgical Management of Abdominal Wall Hernias in adults-epidemiological aspects and our experiences." (2013). Priory Medical Journal. 
11. Zieren MD, J., Zieren MD, H. U., Jacobi MD, C. A., Wenger MD, F. A., \& Müller MD, J. M. (1998). Prospective randomized study comparing laparoscopic and open tension-free inguinal hernia repair with Shouldice's operation. The American Journal of Surgery, 175(4), 330-333.

12. Johansson, Bo, et al. "Laparoscopic mesh versus open preperitoneal mesh versus conventional technique for inguinal hernia repair: a randomized multicenter trial (SCUR Hernia Repair Study)." Annals of surgery 230.2 (1999): 225.

13. Lal Pawanindra, R. K. Kajla, J. Chander, R. Saha, and V. K. Ramteke. "Randomized controlled study of laparoscopic total extraperitoneal versus open Lichtenstein inguinal hernia repair." Surgical endoscopy 17, no. 6 (2003): 850-856.

14. MRC Laparoscopic Groin Hernia Trial Group. Laparoscopic versus open repair of groin hernia: A randomized comparison. Lancet 1999;354:185-190.

15. Picchio M, Lombardi A, Zolovkins A, et al. Tension-free laparoscopic and open hernia repair. Randomized controlled trial of early results.World J Surg 1999;23:1004-1009.

16. Shaikh, M. S., Abro, A. A., Naz, S., Shaikh, S. A., Shaikh, A. G., Seelro, R., \& Bhatti, N. (2009). Outcomes of open mesh hernia repair: five year's experience at Chandka Medical College Hospital Larkana. Jlumhs, 8(3), 205-9.

17. Gianetta, E., Cuneo, S., Vitale, B., Camerini, G., Marini, P., \& Stella, M. (2000). Anterior tension-free repair of recurrent inguinal hernia under local anesthesia: a 7-year experience in a teaching hospital. Annals of surgery, 231(1), 132.

18. Neumayer $\mathrm{L}^{1}$, Giobbie-Hurder A, Jonasson O, Fitzgibbons R Jr, Dunlop D, Gibbs J, Reda D, Henderson W, Open mesh versus laparoscopic mesh repair of inguinal hernia. N Engl J Med. 2004 Apr 29;350(18):1819-27. Epub 2004 Apr 25.

19. Juul, P., and K. Christensen. "Randomized clinical trial of laparoscopic versus open inguinal hernia repair." British journal of surgery 86.3 (1999): 316-319.

20. 138. Bringman, S., Ramel, S., Heikkinen, T. J., Englund, T., Westman, B., \& Anderberg, B. (2003). Tension-free inguinal hernia repair: TEP versus meshplug versus Lichtenstein: a prospective randomized controlled trial. Annals of surgery, 237(1), 142.

21. Bhandarkar, Deepraj S., Manu Shankar, and Tehemton E. Udwadia. "Laparoscopic surgery for inguinal hernia: Current status and controversies."Journal of minimal access surgery 2, no. 3 (2006): 178.

22. Erhan, Yamac, Elvan Erhan, Hasan Aydede, Metin Mercan, and Demet Tok. "Chronic pain after Lichtenstein and preperitoneal (posterior) hernia repair."Canadian Journal of Surgery 51, no. 5 (2008): 383.

23. Poobalan, Amudha S., Julie Bruce, W. Cairns S. Smith, Peter M. King, Zygmunt H. Krukowski, and W. Alastair Chambers. "A review of chronic pain after inguinal herniorrhaphy." The Clinical journal of pain 19, no. 1 (2003): 48-54.

24. Heikkinen, Timo J., Kari Haukipuro, and A. Hulkko. "A cost and outcome comparison between laparoscopic and Lichtenstein hernia operations in a daycase unit." Surgical endoscopy 12, no. 10 (1998): 1199-1203.

25. Wilson, M. S., G. T. Deans, and W. A. Brough. "Prospective trial comparing Lichtenstein with laparoscopic tension-free mesh repair of inguinal hernia. "British journal of surgery 82, no. 2 (1995): 274277.

26. Liem, Mike SL, et al. "Comparison of conventional anterior surgery and laparoscopic surgery for inguinal-hernia 
repair." New England Journal of

Medicine 336.22 (1997): 1541-1547.

27. Andersson B, Hallen M, Leveau P, et al.

Laparoscopic extraperitoneal inguinal hernia repair versus open mesh repair. A prospective randomized controlled trial. Surgery 2003;133:464-472.

28. Stoker DL, Spiegelhalter DJ, Singh R, et al. Laparoscopic versus open inguinal hernia repair. Randomised prospective trial. Lancet 1994;343:1243-1245.

29. Feliu, X., R. Claveria, P. Besora, J. Camps, E. Fernández-Sallent, X. Viñas, and J. M. Abad. "Bilateral inguinal hernia repair: laparoscopic or open approach?." Hernia 15, no. 1 (2011): 15-18.

30. Gokalp A, Inal M, Maralcan G, Baskonus I. A prospective randomized study of Lichtenstein open tension-free versus laparoscopic totally extraperitoneal techniques for inguinal hernia repair. Acta Chir Belg 2003; 103:502-6.

31. Langeveld HR, van't Riet M, Weidema WF, Stassen LPS, Steyerberg EW, Lange $\mathrm{J}$, et al. Total extraperitoneal inguinal hernia repair compared with Lichtenstein (the LEVEL-Trial): a randomized controlled trial. Ann Surg 2010; 251:819-24.

32. Eklund A, Carlsson P, Rosenblad A, Montgomery A, Bergkvist L, Rudberg C, et al. Long-term cost-minimization analysis comparing laparoscopic with open (Lichtenstein) inguinal hernia repair. Br J Surg 2010; 97:765-71. 